\title{
Strong Consistency of the Spline-Estimation of Probabilities Density in Uniform Metric
}

\author{
Mukhammadjon S. Muminov', Khaliq S. Soatov ${ }^{2}$ \\ ${ }^{1}$ Institute of Mathematics, National University of Uzbekistan, Tashkent, Uzbekistan \\ ${ }^{2}$ Tashkent University of Information Technologies, Tashkent, Uzbekistan \\ Email:m.muhammad@rambler.ru, kh.soatov@mail.ru
}

Received 5 December 2015; accepted 24 April 2016; published 27 April 2016

Copyright (C) 2016 by authors and Scientific Research Publishing Inc.

This work is licensed under the Creative Commons Attribution International License (CC BY).

http://creativecommons.org/licenses/by/4.0/

(c) (i) Open Access

\section{Abstract}

In the present paper as estimation of an unknown probability density of the spline-estimation is constructed, necessity and sufficiency conditions of strong consistency of the spline-estimation are given.

\section{Keywords}

Strong Consistency, Spline-Estimation, Probability Density in Uniform Metric, Uniform Metric, Soatov, Muminov, Tashkent University, Institute of Mathematics

\section{Introduction}

We assume that on the interval $[a, b], a, b \in(-\infty,+\infty), a<b$. The following mesh

$$
\Delta_{N}: a=x_{0}<x_{1}<\cdots<x_{N}=b \text {, }
$$

is given, where $N$ is a natural number. Let $P_{k}$ be the set of polynomials of degree $\leq k$ and $C_{k}[a, b]$ be the set of continuous on the $[a, b]$ functions having continuous derivative of order $k, k=1,2, \cdots$. In the book of Stechkin and Subbotin [1] the following is given.

Definition. The function $S_{N}(x)=S_{N}(x, F)$ is called by interpolation cubic spline with respect to the mesh (1) for the function $F(x)$, if:

a) $S_{N}(x) \in P_{3}, x \in\left[x_{i-1}, x_{i}\right], i=\overline{1, N}$,

b) $S_{N}(x) \in C_{2}[a, b]$,

c) $S_{N}\left(x_{i}\right)=t_{i}=\overline{0, N}, N \geq 2$.

Here $t_{i}=F\left(x_{i}\right) \cdot i=\overline{0, N}$.

The points $\left\{x_{i}\right\}$ are called by the nodes of the spline. 
Later on for convenience we let $[a, b]=[0,1]$ and the obtained results will remain valid for any finite interval $[a, b]$.

Let $X_{1}, X_{2}, \cdots, X_{N}$ be independent identical distributed random variables with unknown density distribution $f(x)$ concentrated and continuous on the interval $[0,1]$, and $S_{N}(x)$ be cubic spline interpolating the values $y_{k}=F_{n}\left(x_{k}\right)$ in the points $x_{k}=k h, k=\overline{0, N}, N=N_{(n)}$ with "boundary conditions"

$$
S_{N}^{\prime}(a)=a_{N}, S_{N}^{\prime}(b)=b_{N} .
$$

Here $F_{n}(x)$ is the empirical function of the distribution of the sample $X_{1}, X_{2}, \cdots, X_{N}, h=\frac{1}{N}$ and $n h \rightarrow \infty$, $h \rightarrow 0$ as $n \rightarrow \infty, a_{N}$ and $b_{N}$ are given real numbers. Concrete choice of these numbers depends on the considered problem.

As estimation of an unknown probability density we take the statistics $S_{N}^{\prime}(x)$.

In the present work as estimation of the unknown density $f(x)$ we take the statistics $S_{n}^{\prime}(x)$ defined as in Theorem 1 and in Theorem 2 as well.

It is clear that, in Theorems 1 and 2 spline estimations are constructed with different boundary conditions.

Theorem 3 is devoted to asymptotic unbiasedness of the spline estimation. Also for completeness of the results the dispersion and the covariance of the spline-estimation are given.

In the main Theorem 4 necessity and sufficiency conditions for strong consistency of the spline-estimation are given.

Similar result for the Persen-Rozenblatt estimation is obtained in the book of Nadaraya (1983) [2].

More detailed review on spline estimation is given in works of Wegman, Wright [3], Muminov [4].

\section{Auxiliary Results}

Using the results of the work Lii [5] the following theorems are easily proved.

\subsection{Theorem 1}

Let $F_{n}(x)$ be empirical function of the distribution constructed by simple sample $X_{1}, X_{2}, \cdots, X_{N}$ and $S_{N}(x)$ be cubic spline interpolating the values $F_{n}\left(x_{k}\right)$ in the nodes of the mesh (1). If we choose the boundary conditions for $S_{N}(x)$ in the form

$$
a_{N}=\frac{y_{1}-y_{0}}{h}, b_{N}=\frac{y_{N}-y_{N-1}}{h}
$$

then the derivative $S_{N}^{\prime}(x)$ of the spline function is defined by the equality

$$
S_{N}^{\prime}(x)=\frac{1}{h} \int_{0}^{1} W_{N}(x, y) \mathrm{d} F_{N}(y) .
$$

Here $W_{N}(x, y)=W_{N, i, j}(x, y)=E_{i, j}(x)$, for $x \in\left[x_{i-1}, x_{i}\right], y \in\left[x_{j}, x_{j+1}\right], i=\overline{0, N-1}, 0$

$$
E_{i, j}(x)=\left\{\begin{array}{l}
D_{i, j}(x), \quad j \neq i-1 \\
D_{i, j}(x)+1, \quad j=i-1
\end{array}\right.
$$

and

$$
D_{i, j}(x)= \begin{cases}-\frac{3}{2} C_{i, 1}(x) & j=0, \\ \frac{3}{2}\left[C_{i, j}(x)-C_{i, j+1}(x)\right], & j=1,2, \cdots, N-2, \\ \frac{3}{2} C_{i, N-1}(x) & j=N-1 .\end{cases}
$$

$C_{i, j}(x)$ are defined by the following relations: 


$$
\begin{gathered}
C_{i, j}(x)=A_{i-1, j}^{-1}\left\lceil\frac{1}{3}-(1-r)^{2}\right\rceil+A_{i-1, j}^{-1}\left(r^{2}-\frac{1}{3}\right), \\
r=\frac{x-x_{i-1}}{h}, i=\overline{1, N}, j=\overline{0, N-1},
\end{gathered}
$$

where

$$
\begin{gathered}
A_{i, j}^{-1}=\frac{\sigma^{j-1}\left(1+\sigma^{2 i}\right)\left(1+\sigma^{2 N-2 j}\right)}{(2+\sigma)\left(1-\sigma^{2 N}\right)}, 0<i \leq j<N, \\
A_{i, N}^{-1}=\frac{\sigma^{N-i}\left(1+\sigma^{2 i}\right)}{(2+\sigma)\left(1-\sigma^{2 N}\right)}, 0<i \leq N, \\
A_{0, j}^{-1}=\frac{2 \sigma^{j}\left(1+\sigma^{2 N-2 j}\right)}{(2+\sigma)\left(1-\sigma^{2 N}\right)}, 0<j<N, \\
A_{0, N}^{-1}=\frac{2 \sigma^{N}}{(2+\sigma)\left(1-\sigma^{2 N}\right)}, \quad A_{0,0}^{-1}=\frac{2-\sigma^{N-1}(1+\sigma)^{2}}{2(2+\sigma)\left(1-\sigma^{2 N}\right)}, \\
\sigma=\sqrt{3}-2, A_{i, j}^{-1}=A_{N-1, N-j}^{-1} \text { for the other } i \text { and } j .
\end{gathered}
$$

\subsection{Theorem 2}

Let $F_{n}(x)$ be empirical function of the distribution constructed by simple sample $X_{1}, X_{2}, \cdots, X_{n}$ and $S_{N}(x)$ be cubic spline interpolating the values $F_{n}\left(x_{k}\right)$. in the mesh (1). If we choose the boundary conditions for $S_{N}(x)$ in the form

$$
\begin{gathered}
\alpha_{N}=\frac{1}{h}\left(\frac{1}{3} y_{3}-\frac{3}{2} y_{2}+3 y_{1}-\frac{11}{6} y_{0}\right), \\
b_{N}=\frac{1}{h}\left(\frac{11}{6} y_{N}-3 y_{N-1}+\frac{3}{2} y_{N-2}-\frac{1}{3} y_{N-3}\right) .
\end{gathered}
$$

Then the derivative $S_{N}^{\prime}(x)$ of the spline function is defined by the equality

$$
S_{N}^{\prime}(x)=\frac{1}{h} \int_{0}^{1} W_{N}(x, y) \mathrm{d} F_{n}(y),
$$

where $W_{N}(x, y)=W_{N / i, j}(x, y)=\widehat{E_{i, j}}(x)$, for $x \in\left[x_{i-1}, x_{i}\right], y \in\left[x_{j}, x_{j+1}\right]$,

$$
\begin{aligned}
& i=\overline{0, N-1}, \\
& \hat{E}_{i, j}(x)= \begin{cases}\hat{D}_{i, j}(x) & j \neq i-1 \\
\hat{D}_{i, j}(x)+1 & j=i-1\end{cases} \\
& \hat{D}_{i, 0}=-\frac{3}{2} C_{i, 1}-\frac{5}{2} C_{i, 0}, \quad \hat{D}_{i, 1}=\frac{3}{2}\left(C_{i, 1}-C_{i, 2}\right)+\frac{7}{2} C_{i, 0}, \\
& \hat{D}_{i, 2}=\frac{3}{2}\left(C_{i, 2}-C_{i, 3}\right)-C_{i, 0}, \quad \hat{D}_{i, j}=\frac{3}{2}\left(C_{i, j}-C_{i, j+1}\right), j=3,4, \cdots, N-4, \\
& \hat{D}_{i, N-3}=\frac{3}{2}\left(C_{i, N-3}-C_{i, N-2}\right)+C_{i, N}, \quad \hat{D}_{i, N-2}=\frac{3}{2}\left(C_{i, N-2}-C_{i, N-1}\right)-\frac{7}{2} C_{i, N}, \\
& \hat{D}_{i, N-1}=\frac{3}{2} C_{i, N-1}+\frac{5}{2} C_{i, N},
\end{aligned}
$$


and $C_{i, j}$ are defined by formula (2).

We introduce the following denotations:

$X_{1}, X_{2}, \cdots, X_{n}$ is the simple sample from the general population

$$
F(t)=\int_{-\infty}^{t} f(x) \mathrm{d} x ;
$$

$F_{n}^{*}(t)=F_{n}\left(F^{-1}(t)\right)$ is empirical function of distribution of the sample $F\left(X_{1}\right), F\left(X_{2}\right), \cdots, F\left(X_{n}\right)$;

$Y_{n}(t)=\sqrt{n}\left[F_{n}^{*}(t)-t\right], t \in[0,1]$ is the empirical process;

$\left\{\omega_{n}(t), t \in[0,1]\right\}$ is the sequence of wiener processes;

$B_{n}(t)=\omega_{n}(t)-t \omega_{n}(1), t \in[0,1]$ is the brownian bridge.

We give the auxiliary lemmas.

\subsection{Lemma 1 [6]}

There exists a probability space $(\Omega, F, P)$.

On which it can be defined version $F_{n}^{*}(t)$ and the sequence of Brownian bridges $B_{n}(t)$ such that for all $x>0$

$$
P\left(\sup _{0 \leq t \leq 1}\left|n\left(F_{n}^{*}(t)-t\right)-\sqrt{n} B_{n}(t)\right|>a x+b \log n+c \log 2\right) \leq \mathrm{e}^{-x},
$$

where $a=3.26, b=4.86, c=2.70$.

\subsection{Lemma 2 [7]}

Let $\omega$ be modulus of continuity of the brownian bridge $B_{n}(t)$,

$$
p(u)= \begin{cases}\sqrt{u(1-u),} & \text { if } 0 \leq u \leq 1 / 2, \\ 1 / 2, & \text { if } u>1 / 2\end{cases}
$$

and $q(u)=\int_{0}^{u} \sqrt{\ln (1 / v)} \mathrm{d} p(v)$. Then with probability $1 \omega$ does not exceed the quantity $16\left(p \sqrt{\ln v_{\varepsilon}}+q \sqrt{2}\right)$. Here $v_{\varepsilon}$ is the random variable which is not less than 1 almost everywhere and $M v_{\varepsilon}<4 \sqrt{2}$.

\section{Main Results and Proofs}

The following theorem characterizes the asymptotic behavior of the bias, the covariance and the dispersion of the spline estimation.

\subsection{Theorem 3}

Let $S_{N}^{\prime}(x)$ be the spline estimation.

1) If $f \in C_{k}[0,1], k=0,1,2$ and $S_{N}^{\prime}(x)$ are defined as in Theorem 2, then for $n \rightarrow \infty$

$$
M S_{N}^{\prime}(x)=f(x)+o\left(h^{k}\right) .
$$

2) If $f \in C[0,1]$ and $S_{N}^{\prime}(x)$ are defined as in Theorem 1 , then

$$
\begin{gathered}
\sup _{0 \leq x \leq 1}\left|M S_{N}^{\prime}(x)-f(x)\right| \rightarrow 0, n \rightarrow \infty, \\
D S_{N}^{\prime}(x)=\frac{f(x)}{n h} A(r)+O(h / n), n \rightarrow \infty,
\end{gathered}
$$

where $0<x<1$, 


$$
\begin{aligned}
& A(r)=-\frac{3(1-\sigma)}{2+\sigma}\left(2 r^{2}-2 r+\frac{1}{3}\right)+\frac{9}{4}\left(\frac{1-\sigma}{2+\sigma}\right)^{2} \\
& \times\left\{\left(2 r^{2}-2 r+\frac{1}{3}\right)^{2}+\left[\left(r^{2}-\frac{1}{3}\right)+\sigma\left(\frac{1}{3}-(1-r)^{2}\right)\right]^{2} \frac{1}{1-\sigma^{2}}\right. \\
&\left.+\left[\left(r^{2}-\frac{1}{3}\right)+\frac{1}{\sigma}\left(\frac{1}{3}-(1-r)^{2}\right)\right]^{2} \frac{\sigma^{2}}{1-\sigma^{2}}\right\}, \\
& \sigma=\sqrt{3}-2, r=\frac{x-x_{i-1}}{h}, x_{i-1}=\frac{\llbracket N_{x} \rrbracket}{N},
\end{aligned}
$$

$[y]$ is the integer part of the number $y$.

3) Suppose $0<x, y<1, \quad x_{i-1}=\frac{\llbracket N_{x} \rrbracket}{N}, x_{j-1}=\frac{\llbracket N_{y} \rrbracket}{N}, d=i-j, \quad r=\frac{x-x_{i-1}}{h}$ and $r_{2}=\frac{y-x_{j-1}}{h}$, then for $n \rightarrow \infty$

$$
\begin{aligned}
& \operatorname{cov}\left[S_{N}^{\prime}(x), S_{N}^{\prime}(y)\right] \\
& =\frac{1}{n h} \cdot \frac{3}{4} f(x)\left\{\left[\left(r_{1}^{2}-\frac{1}{3}\right)\left(r_{2}^{2}-\frac{1}{3}\right)+\left(\frac{1}{3}-\left(1-r_{1}\right)^{2}\left(\frac{1}{3}-\left(1-r_{2}\right)\right)^{2}\right)\right]\left(6|d| \sigma^{|d|}-\frac{12 \sigma^{|d+1|}}{1-\sigma^{2}}\right)\right. \\
& +\left(r_{1}^{2}-\frac{1}{3}\right)\left(\frac{1}{3}-\left(1-r_{2}\right)^{2}\right)\left(6|d+1| \sigma^{|d+1|}-\frac{12 \sigma^{|d+1|+1}}{1-\sigma^{2}}\right) \\
& \left.+\left(r_{2}^{2}-\frac{1}{3}\right)\left(\frac{1}{3}-\left(1-r_{1}\right)^{2}\right)\left(6|d-1| \sigma^{|d-1|}-\frac{12 \sigma^{|d-1|+1}}{1-\sigma^{2}}\right)\right\} \\
& +\frac{f(y)}{n h} \cdot \frac{\sqrt{3}}{2}\left[\left(r_{1}^{2}-\frac{1}{3}\right)\left(\sigma^{|d+1|}-\sigma^{|d|}\right)+\left(\frac{1}{3}-\left(1-r_{1}\right)^{2}\right) \times\left(\sigma^{|d|}-\sigma^{|d-1|}\right)\right] \\
& +\frac{f(x)}{n h} \cdot \frac{\sqrt{3}}{2}\left[\left(r_{2}^{2}-\frac{1}{3}\right)\left(\sigma^{|d-1|}-\sigma^{|d|}\right)+\left(\frac{1}{3}-\left(1-r_{2}\right)^{2}\right)\left(\sigma^{|d|}-\sigma^{|d+1|}\right)\right]+\frac{f(x)}{n h} \delta_{d, 0}+0\left(\frac{1}{n}\right) .
\end{aligned}
$$

Proof. By virtue of $M S_{N}^{\prime}(x)=\left(M S_{N}^{\prime}(x)\right)^{\prime}$, Theorems 9, 11, 12 from Stechkin and Subbotin [1] and Theorems 1 from Lii [5] follows the first statement of Theorem 3. The second and the third statement of Theorem 3 are proved in Lii [5].

\subsection{Theorem 4}

Suppose $\frac{\ln n}{n h} \rightarrow 0$ as $n \rightarrow \infty$. Then in order with probability 1

$$
\sup _{0 \leq x \leq 1}\left|S_{N}^{\prime}(x)-g(x)\right| \rightarrow 0 \text { as } n \rightarrow \infty,
$$

it is necessary and sufficient that the function $g(x)$ is the density of the distribution $F(x)$ concentrated and continuous on the interval $[0,1]$ with respect to Lebesgue measure.

Proof. Sufficiency. It is clear that

$$
\sup _{0 \leq x \leq 1}\left|S_{N}^{\prime}(x)-f(x)\right| \leq \varepsilon_{N}+\delta_{N},
$$

where 


$$
\varepsilon_{N}=\sup _{0 \leq x \leq 1}\left|S_{N}^{\prime}(x)-M S_{N}^{\prime}(x)\right|, \quad \delta_{N}=\sup _{0 \leq x \leq 1}\left|M S_{N}^{\prime}(x)-f(x)\right| .
$$

First we estimate the term $\varepsilon_{N}$ in the right hand part of (3). We have

$$
\varepsilon_{N} \leq \frac{32}{\sqrt{n h}}\left[\sup _{0 \leq x \leq 1}\left|Y_{n}(t)-B_{n}(t)\right|+\frac{1}{2} \max _{1 \leq i \leq N}\left|B_{n}\left(F\left(x_{i}\right)\right)-B_{n}\left(F\left(x_{i-1}\right)\right)\right|\right] .
$$

From Lemma 1 it follows that with probability 1 for $n \rightarrow \infty$

$$
\sup _{0 \leq x \leq 1}\left|Y_{n}(t)-B_{n}(t)\right|=0\left(\frac{\ln n}{\sqrt{n}}\right) .
$$

If we denote the modulus of continuity $B_{n}(t)$ by $\theta(h)$ then from

Lemma 2

$$
\left|B_{n}\left(F\left(x_{i}\right)\right)-B_{n}\left(F\left(x_{i-1}\right)\right)\right| \leq(1+B) \theta(h)
$$

where

$$
\begin{gathered}
B=\sup _{0 \leq t \leq 1} f(t) \\
\theta(h) \leq 16 \sqrt{h}\left[\sqrt{\ln v_{n}}+\sqrt{2}(\sqrt{\ln N}+\sqrt{2 \pi / \ln 2})\right],
\end{gathered}
$$

with probability $1 v_{n} \geq 1$ and $M v_{n}<4 \sqrt{2}$.

This, combining (3)-(6) and using Theorem 3 we get the sufficiency condition of Theorem 4 .

Necessity. Let with probability 1

$$
\sup _{0 \leq x \leq 1}\left|S_{N}^{\prime}(x)-g(x)\right| \rightarrow 0 \text { as } n \rightarrow \infty .
$$

Hence, from continuity of $S_{N}^{\prime}(x)$ it follows continuity of $g(x)$ on the interval $[0,1]$.

Therefore, the sequence random variables

$$
\tau_{n}=\sup _{0 \leq x \leq 1}\left|S_{N}^{\prime}(x)-g(x)\right|, n=1,2, \cdots
$$

are uniformly integrable. Therefore according to Theorem 5 from Shiryaev [8] and the inequalities

$$
\begin{aligned}
& \sup _{0 \leq x \leq 1}\left|M S_{N}^{\prime}(x)-g(x)\right|=\sup _{0 \leq x \leq 1}\left|M\left(S_{N}^{\prime}(x)-g(x)\right)\right| \\
& \leq \sup _{0 \leq x \leq 1} M\left|S_{N}^{\prime}(x)-g(x)\right| \leq M \sup _{0 \leq x \leq 1}\left|S_{N}^{\prime}(x)-g(x)\right|
\end{aligned}
$$

it follows that for $n \rightarrow \infty$

$$
\sup _{0 \leq x \leq 1}\left|M S_{N}^{\prime}(x)-g(x)\right| \rightarrow 0 .
$$

By virtue of (7) it is easy to see that the sequence of functions

$$
g_{n}(x)=\frac{1}{h} \int_{0}^{1} W_{N}(x, y) \mathrm{d} F(y)
$$

uniformly converges to some continuous function $g_{0}(x)$, i.e. for $n \rightarrow \infty$

$$
\sup _{0 \leq x \leq 1}\left|g_{n}(x)-g_{0}(x)\right| \rightarrow 0 .
$$

We show now continuity of $F(x)$ on the interval $[0,1]$.

We assume the inverse that there exists a point $x_{0}, x_{0} \in[0,1]$ such that $P\left(X_{1}=x_{0}\right)=p_{0}>0$. Then by virtue of (8) and

$$
\frac{p_{0}}{h} \sup _{0 \leq x \leq 1}\left|W_{n}\left(x, x_{0}\right)\right| \leq \sup _{0 \leq x \leq 1}\left|g_{n}(x)\right| \leq \frac{1}{h} \sup _{0 \leq x \leq 1}\left|W_{n}(x, y)\right|
$$


it follows continuity of $F(x)$ on the interval $[0,1]$.

By (8) for all $0 \leq x, y \leq 1$

$$
\begin{gathered}
\lim _{n \rightarrow \infty} \int_{y}^{x} M S_{N}^{\prime}(t) \mathrm{d} t=\int_{y}^{x} g(t) \mathrm{d} t \\
\int_{y}^{x} M S_{N}^{\prime}(t) \mathrm{d} t=\int_{y}^{x} \mathrm{~d}\left(M S_{N}(t)\right)=M S_{N}(x)-M S_{N}(y) .
\end{gathered}
$$

From another side, according to Theorem 11 from Stechkin and Subbotin (1976)

$$
\lim _{n \rightarrow \infty} M S_{N}(x)=F(x) .
$$

By virtue of (9)-(11)

$$
F(x)-F(y)=\int_{y}^{x} g(t) \mathrm{d} t
$$

Theorem 4 is proved.

\section{References}

[1] Stechkin, S.B. and Subbotin, Y.N. (1976) Splines in Computational Mathematics. Moscow, Nauka, 272 p.

[2] Nadaraya, E.A. (1983) Nonparametric Estimation of Probability Density and Regression Curve. Tbilisi University, Tbilisi, 195 p.

[3] Wegman, E.J. and Wright, I.W. (1983) Splinesin Statistics. Journal of the American Statistical Association, 78, 351365. http://dx.doi.org/10.1080/01621459.1983.10477977

[4] Muminov, M.S. (2010) On Appoximation of the Probability of the Lagre Outlier of Nonstationary Gauss Process. Siberian Mathematical Journal, 51, 175-195. http://dx.doi.org/10.1007/s11202-010-0015-6

[5] Lii, K.S. (1978) A Global Measure of a Spline Density Estimate. Annals of Statistics, 6, 1138-1148. http://dx.doi.org/10.1214/aos/1176344316

[6] Rio, E. (1994) Local Invariance Principles and Application to Density Estimation. Probability Theory and Related Fields, 98, 21-26. http://dx.doi.org/10.1007/BF01311347

[7] Garsia, F. (1970) Continuity Properties of Gaussian Processer with Multidimensional Time Parameter. Proceedings of the Sixth Berkeley Symposium on Mathematical Statistics and Probability, 369-374.

[8] Shiryaev, A.N. (1982) Probability. Moscow, Nauka, 576 p. 